\title{
A SHORT PROOF OF SHÔ ISEKI'S FUNCTIONAL EQUATION
}

\author{
TOM M. APOSTOL
}

1. Introduction. A few years ago Shô Iseki [4] derived the functional equation

$$
\Lambda_{p}(\alpha, \beta, z)=(i z)^{p-1} \Lambda_{p}\left(1-\beta, \alpha, z^{-1}\right)+R_{p}(\alpha, \beta, z),
$$

where

$$
\begin{aligned}
\Lambda_{p}(\alpha, \beta, z) & =\sum_{r=0}^{\infty}\left\{\lambda_{p}((r+\alpha) z-i \beta)+\lambda_{p}((r+1-\alpha) z+i \beta)\right\}, \\
\lambda_{p}(x) & =\sum_{m=1}^{\infty} m^{-p} e^{-2 \pi m x} \quad(\Re(x)>0),
\end{aligned}
$$

and

(3) $\quad R_{p}(\alpha, \beta, z)=-\frac{(2 \pi z)^{p}}{(p+1) !} \sum_{n=0}^{p+1}\left(\begin{array}{c}p+1 \\ n\end{array}\right)(i z)^{-n} B_{p+1-n}(\alpha) B_{n}(\beta)$.

In (3), $B_{n}(t)$ is the Bernoulli polynomial defined by

$$
\frac{z e^{t z}}{e^{z}-1}=\sum_{n=0}^{\infty} \frac{B_{n}(t)}{n !} z^{n} \quad(|z|<2 \pi) .
$$

The parameter $p$ in (1) is required to be a positive odd integer and $z$ is any complex number with $\Re(z)>0$. The parameters $\alpha$ and $\beta$ are real numbers restricted as follows: When $p=1$ we must have $0 \leqq \alpha \leqq 1$ and $0<\beta<1$, or $0<\alpha<1$ and $0 \leqq \beta \leqq 1$. For odd $p>1$, Equation (1) is valid for $0 \leqq \alpha \leqq 1$ and $0 \leqq \beta \leqq 1$.

In the case $p=1$, Iseki's formula (1) can be used to derive the famous functional equation for the Dedekind modular function $\eta(\tau)$. In the case of odd $p>1$, it yields a transformation formula for the Lambert series

$$
G_{p}(x)=\sum_{n=1}^{\infty} n^{-p} \frac{x^{n}}{1-x^{n}} \quad(|x|<1)
$$

which describes the behaviour of $G_{p}\left(e^{2 \pi i r}\right)$ under a modular substitution $\tau^{\prime}=(a \tau+b) /(c \tau+d)$. (See $[4 ; 1]$.)

Received by the editors March 7, 1963. 
Recently Iseki [5] gave a simpler proof of (1) for the case $p=1$, using a revision of Rademacher's Mellin-transform method for treating Dedekind's function $\eta(\tau)$ [6]. In this note we show how the method can be employed to give a short proof of (1) in the general case of any odd $p>1$.

2. The proof. First we assume $0<\alpha<1,0<\beta<1$. Using the Mellin transform

$$
e^{-y}=\frac{1}{2 \pi i} \int_{(3 / 2)} \Gamma(s) y^{-s} d s \quad(\Re(y)>0),
$$

where $\int_{(c)}$ is an abbreviation for $\int_{c-\infty i}^{c+\infty i}$, we find

$$
\sum_{r=0}^{\infty} \lambda_{p}((r+\alpha) z-i \beta)=\frac{1}{2 \pi i} \int_{(3 / 2)} \frac{\Gamma(s)}{(2 \pi z)^{s}} \zeta(s, \alpha) \zeta_{\beta}(p+s) d s,
$$

where

$$
\zeta(s, \alpha)=\sum_{n=0}^{\infty} \frac{1}{(n+\alpha)^{s}}, \quad \zeta_{\beta}(s)=\sum_{n=1}^{\infty} \frac{e^{2 \pi i \beta n}}{n^{*}} .
$$

We remark that both $\zeta(s, \alpha)$ and $e^{-2 \pi i \beta} \zeta_{\beta}(s)$ are special cases of the Lerch zeta function $\phi(x, a, s)$ defined as the analytic continuation of the series

$$
\phi(x, a, s)=\sum_{n=0}^{\infty} \frac{e^{2 n \pi i x}}{(n+a)^{\bullet}} .
$$

(See [2].) From (2) and (4) we obtain

$$
\Lambda_{p}(\alpha, \beta, z)=\frac{1}{2 \pi i} \int_{(3 / 2)} z^{-8} F_{p}(\alpha, \beta, s) d s,
$$

where

(6) $F_{p}(\alpha, \beta, s)=\frac{\Gamma(s)}{(2 \pi)^{s}}\left\{\zeta(s, \alpha) \zeta_{\beta}(p+s)+\zeta(s, 1-\alpha) \zeta_{1-\beta}(p+s)\right\}$.

If we shift the line of integration in (5) from $\Re(s)=3 / 2$ to $\Re(s)$ $=-p-1 / 2$ we obtain

$$
\Lambda_{p}(\alpha, \beta, z)=\frac{1}{2 \pi i} \int_{(-p-1 / 2)} z^{-\triangleleft} F_{p}(\alpha, \beta, s) d s+R,
$$

where $R$ is the sum of residues introduced when the path crosses the poles of the integrand. The displacement of the path is easily justified 
as in Iseki's paper. In (7) we make the change of variable $u=1-p-s$ to get

$$
\Lambda_{p}(\alpha, \beta, z)=\frac{z^{p-1}}{2 \pi i} \int_{(3 / 2)} z^{u} F_{p}(\alpha, \beta, 1-p-u) d u+R .
$$

Next we prove that we have

$$
F_{p}(\alpha, \beta, 1-p-s)=i^{p-1} F_{p}(1-\beta, \alpha, s) .
$$

For this purpose we return to (6) and express $F_{p}(\alpha, \beta, s)$ entirely in terms of Hurwitz zeta functions, using the formula

$$
\begin{aligned}
\zeta_{\beta}(p+s)=\Gamma(1-p-s)(2 \pi)^{p+s-1}\{ & e^{\pi i(1-p-s) / 2} \zeta(1-p-s, \beta) \\
& \left.+e^{\pi i(p+s-1) / 2} \zeta(1-p-s, 1-\beta)\right\}
\end{aligned}
$$

(see $[5$, equation (3) $]$ ) and a corresponding formula for $\zeta_{1-\beta}(p+s)$. We find

$$
\begin{aligned}
& F_{p}(\alpha, \beta, s)=(2 \pi)^{p-1} \Gamma(s) \Gamma(1-p-s) \\
& \quad \cdot\left\{e^{\pi i(1-p-s) / 2}[\zeta(s, \alpha) \zeta(1-p-s, \beta)+\zeta(s, 1-\alpha) \zeta(1-p-s, 1-\beta)]\right. \\
& +e^{-\pi i(1-p-s) / 2}[\zeta(s, \alpha) \zeta(1-p-s, 1-\beta) \\
& \quad+\zeta(s, 1-\alpha) \zeta(1-p-s, \beta)]\} .
\end{aligned}
$$

If we replace $\alpha$ by $1-\beta, \beta$ by $\alpha$, and $s$ by $1-p-s$, this formula becomes

$$
\begin{aligned}
& F_{p}(1-\beta, \alpha, 1-p-s)=(2 \pi)^{p-1} \Gamma(1-p-s) \Gamma(s) \\
& \cdot\left\{e^{\pi i s / 2}[\zeta\right.(1-p-s, 1-\beta) \zeta(s, \alpha) \\
&+\zeta(1-p-s, \beta) \zeta(s, 1-\alpha)] \\
&+e^{-\pi i s / 2}[\zeta(1-p-s, 1-\beta) \zeta(s, 1-\alpha) \\
&+\zeta(1-p-s, \beta) \zeta(s, \alpha)]\} .
\end{aligned}
$$

At this point we use the assumption that $p$ is an odd integer to write

$$
e^{\pi i s / 2}=i^{p-1} e^{-\pi i(1-p-s) / 2}, \quad e^{-\pi i s / 2}=i^{p-1} e^{\pi i(1-p-s) / 2}
$$

and we see that (10) and (11) give us (9). Using (9) in the integral in (8) we obtain

$$
\Lambda_{p}(\alpha, \beta, z)=(i z)^{p-1} \Lambda_{p}\left(1-\beta, \alpha, z^{-1}\right)+R .
$$

Therefore, to complete the proof of (1) we need only show that the residue sum $R$ is equal to the right member of (3).

To compute $R$ we use the formula for $F(\alpha, \beta, s)$ given in (6) and we note that the integrand has a simple pole at $s=1$ arising from the 
factor $\zeta(s, \alpha)$ and simple poles at the points $s=0,-1,-2, \cdots,-p$, arising from the factor $\Gamma(s)$. The function $\zeta_{\beta}(s)$ is an entire function of $s$. (See [2].) If $R(k)$ denotes the residue at $s=k$ we have

$$
R(1)=\frac{1}{2 \pi z}\left\{\zeta_{\beta}(p+1)+\zeta_{1-\beta}(p+1)\right\}=\frac{1}{2 \pi z} \sum_{n=-\infty ; n \neq 0}^{\infty} \frac{e^{2 \pi i \beta n}}{n^{p+1}}
$$

whereas for $k=0,1, \cdots, p$ we have

$$
R(-k)=\frac{(-1)^{k}}{k !(2 \pi z)^{-k}}\left\{\zeta(-k, \alpha) \zeta_{\beta}(p-k)+\zeta(-k, 1-\alpha) \zeta_{1-\beta}(p-k)\right\} .
$$

Since $\zeta(-k, \alpha)=-B_{k+1}(\alpha) /(k+1)=(-1)^{k} B_{k+1}(1-\alpha) /(k+1)$ $=(-1)^{k+1} \zeta(-k, 1-\alpha)$ we obtain for $k=0,1, \cdots, p-1$,

$$
\begin{aligned}
R(-k) & =\frac{(-1)^{k+1} B_{k+1}(\alpha)}{(k+1) !(2 \pi z)^{-k}}\left\{\zeta_{\beta}(p-k)+(-1)^{k+1} \zeta_{1-\beta}(p-k)\right\} \\
& =\frac{(-1)^{k+1} B_{k+1}(\alpha)}{(k+1) !(2 \pi z)^{-k}} \sum_{n=1}^{\infty} \frac{e^{2 \pi i \beta n}+(-1)^{k+1} e^{-2 \pi i \beta n}}{n^{p-k}} .
\end{aligned}
$$

Since $p$ is an odd integer the last sum is equal to

$$
\sum_{n=-\infty ; n \neq 0}^{\infty} \frac{e^{2 \pi i \beta n}}{n^{p-k}}=-\frac{(2 \pi i)^{p-k}}{(p-k) !} B_{p-k}(\beta)
$$

(see $[3$, p. 503]), so we have

$$
R(-k)=\frac{(-1)^{k} B_{k+1}(\alpha)(2 \pi i)^{p-k} B_{p-k}(\beta)}{(k+1) !(2 \pi z)^{-k}(p-k) !} .
$$

From (13 and (9) we see that (14) holds also for $k=-1$ and $k=p$, respectively. Therefore we have

$$
\begin{aligned}
R & =\sum_{k=-1}^{p} R(-k)=\sum_{n=0}^{p+1} R(n-p) \\
& =-\frac{(2 \pi z)^{p}}{(p+1) !} \sum_{n=0}^{p+1}\left(\begin{array}{c}
p+1 \\
n
\end{array}\right)(i z)^{-n} B_{p+1-n}(\alpha) B_{n}(\beta) .
\end{aligned}
$$

When this is combined with (12) we get (1). This proves Iseki's functional equation for $0<\alpha<1,0<\beta<1$. Its validity for $\alpha=0,1$ and $\beta=0,1$ when $p>1$ can then be shown as in the original Iseki paper [4].

\section{BiBLIOGRAPHY}

1. T. M. Apostol, Generalized Dedekind sums and transformation formulae of certain Lambert series, Duke Math J. 17 (1950), 147-157. 
2. - On the Lerch zeta function, Pacific J. Math 1 (1951), 161-167.

3. - Mathematical analysis, Addison-Wesley, Reading, Mass., 1957.

4. Shô Iseki, The transformation formula for the Dedekind modular function and related functional equations, Duke Math. J. 24 (1957), 653-662.

5. - A proof of a functional equation related to the theory of partitions, Proc. Amer. Math. Soc. 12 (1961), 502-505.

6. H. Rademacher, Zur Theorie der Modulfunktionen, J. Reine Angew. Math. 167 (1932), 312-336.

California Institute of Technology

\section{ON THE HOMOTOPY CLASSES OF SELF-MAPPINGS OF BORDERED RIEMANN SURFACES}

\section{CLIFFORD J. EARLE ${ }^{1}$}

1. Let $W$ be a Riemann surface which can be represented as the interior of a bordered surface $W_{0}$ with border $B$. Let $F: W \rightarrow W$ be a mapping which has continuous boundary values in $B$. Then $F$ can be extended by symmetry to a self-mapping $F_{*}$ of the doubled surface $W_{*}$. Let the given mapping $F$ be homotopic to the identity map of $W$ onto itself. It is natural to ask whether $F_{*}$ is homotopic to the identity also. In this note we give an affirmative answer for a large number of cases.

2. To be specific we assume that the universal covering surface of $W$ is the disk $U=\{z:|z|<1\} . W$ is of the form $W=U / G$ where $G$ is a Fuchsian group of linear transformations on $U$. $G$ is a group of the second kind; that is, the region of discontinuity $\Omega$ of $G$ contains points on the circumference $\partial U$ of $U . W$ is thus the interior of the bordered surface $W_{0}$ whose border $B$ corresponds to $\Omega \cap \partial U$. The double of $W_{0}$ is the surface $W_{*}=\Omega / G$. We assume that $G$ has at least three limit points, so that the universal covering surface of $W_{*}$ and $\Omega$ is the disk.

It is well known [1, pp. 98-99] that every mapping $F: W \rightarrow W$ is induced by a mapping $f: U \rightarrow U$. That is, $F \phi=\phi f$ where $\phi: U \rightarrow W$ is the natural projection. Moreover, $F$ is homotopic to the identity mapping of $W$ onto $W$ if and only if $f$ can be so chosen that $A f=f A$ for all $A$ in $G$.

Received by the editors March 25, 1963.

1 This research was supported in part by the Air Force Office of Scientific Research. 